\title{
Optimum Extraction of Polysaccharides From Fruiting Body of Catathelasma ventricosum Using Response Surface Methodology
}

\author{
Siqi Zeng ${ }^{1}$, Yiwen $\mathrm{Li}^{1}$, Di Chen ${ }^{1}$, Qianqian Tang ${ }^{1}$, Yuxian You ${ }^{1}$, Aiping Liu ${ }^{1}$, \\ Chaohui Feng ${ }^{1}$, Cheng Li ${ }^{1}$, Yuntao Liu ${ }^{1,2, a^{*}}$ \\ ${ }^{1}$ College of Food Science, Sichuan Agricultural University, Yaan 625014, China. \\ ${ }^{2}$ Animal Nutrition Institute, Sichuan Agricultural University, Chengdu 611130, China. \\ a*lyt_taotao@163.com (corresponding author)
}

Keywords: RSM, Catathelasma ventricosum, Polysaccharides, Extraction, Optimization

\begin{abstract}
Response Surface Methodology (RSM) is employed to optimize the polysaccharides extraction technique of Catathelasma ventricosum. This experiment adopts extraction temperature, extraction time and feed liquid ratio as the influencing factors. The polysaccharides extraction amount of Catathelasma ventricosum and their biological activity are adopted as the response value. According to the combination test design of Box-Benhnken, RSM featuring three factors and three surfaces is adopted to obtain a ternary quadratic linear regression equation. Under the prerequisite of maximizing the biological activity, the optimal extraction conditions after the fitting analysis of the equation are obtained: extraction temperature: $85^{\circ} \mathrm{C}$; extraction time: $3 \mathrm{~h}$; ratio of material to liquid: 0.05 . Under these conditions, the extraction of polysaccharides is about $7.95 \mathrm{~g}$. Besides, results suggest that the actually-measured value stays extremely close to the predicted value. Ultimately proved using RSM is accurate and effective to optimize polysaccharides extraction condition of Catathelasma ventricosum.
\end{abstract}

\section{Introduction}

Catathelasma ventricosum (Peck) Sing is mainly distributed in areas, including Sichuan, Yunnan,Guizhou, Heilongjiang, Tibet, etc. [1-3] By report, Catathelasma ventricosum polysaccharides have good anti-oxidant, anti-tumor, blood fat-reducing and blood pressure-reducing. Therefore, further research into the polysaccharides extraction technique is extremely necessary.

Currently, there have been multiple polysaccharides extraction methods of the edible mushrooms. Water solution extraction is commonly used, including cold water or boiling water extraction, weak acid extraction, weak base or strong base extraction and extraction of some buffer solution, etc. [4-8] Though the extraction methods are diversified, different polysaccharides extraction methods and different reagents can influence structure and biological activity of polysaccharides differently. [9]

As an optimal method, RSM can quickly conduct a comprehensive research into the chosen experimental parameters. In order to increase the polysaccharides production of Catathelasma ventricosum and reduce the influence on the structure of polysaccharides, this paper adopts the pure water as the extraction liquid, and RSM to compare the effects of different extraction temperature, extraction time and ratio of material to liquid on the extraction of polysaccharide and antioxidant activity in vitro. Finally, obtain the optimal extraction method.

\section{Materials and Methods}

Main Reagents. 1,1-Diphenyl-2-picrylhydrazyl(DPPH); 2,6-di-tert-butyl-4-methylphenol(BHT); Ethylenediaminetetraacetic acid (EDTA); dimethyl sulphoxide (DMSO). In the experiment, all reagents and drugs used are above the analysis purity level. All the water used is the ultrapure water and the specific resistance is $18.2 \mathrm{M} \Omega \bullet \mathrm{cm}$.

Materials. Please refer to research methods in the former part as to the picking and pre-treatment of Catathelasma ventricosum [10]. 
Pre-treatment of Materials. Put $375 \mathrm{~g}$ of lyophilized Catathelasma ventricosum into the petroleum ether, shake it in the warm bath for $5 \mathrm{~h}$ within the temperature of $30-60^{\circ} \mathrm{C}$. After centrifuge $(5,000 \mathrm{rpm} ; 10 \mathrm{~min})$, the coarse materials with fat removed are obtained. Use $80 \%$ of ethyl alcohol to wash twice so as to remove the pigment materials, oligosaccharides and other small molecules. Then , the rotary evaporator is used to concentrate and remove the remaining organic solvents. After freeze-drying, materials are kept in the refrigerator at the temperature of $-80^{\circ} \mathrm{C}$.

Optimization Design of Polysaccharides Extraction Technique of Catathelasma ventricosum Fruiting Body. According to the optimization experiment of the polysaccharides extraction technique, extraction temperature, extraction time and ratio of material to liquid are the most obvious factors to the polysaccharides extraction efficiency and its biological activity. [11, 12] Therefore, this research program adopts Box-Benhken design to optimize the extraction temperature, extraction time and ratio of material to liquid. The three factors take three levels, respectively. After the experiment is conducted according to the experiment design table, the data undergo the quadratic regression and fitting to obtain quadratic equation with one-degree term, quadratic term and interaction term. The main effect and interaction effect of various factors are analyzed. In consideration of the extraction efficiency, the extraction method with the highest biological activity is chosen. The quadratic multi-term equation can be expressed below:

$$
\mathrm{Y}=\sum A_{0}+\sum_{i=1}^{3} A_{i} X_{i}+\sum_{i=1}^{3} A_{i i} X_{1}^{2}+\sum_{i=1}^{2} \sum_{j=i+1}^{3} A_{i j} X_{i} X_{j}
$$

$Y$ : Response value;

$A_{0}, A_{i}, A_{i i}$ and $A_{i j}$ : constant, one-degree term, quadratic term and interaction term;

$\mathrm{X}_{\mathrm{i}}$ and $\mathrm{X}_{\mathrm{j}}$ : Independent variables.

Variables and data range of Box-Behnken design are shown in Table 1.

Table 1 Data range and variables of Box-Behnken design

\begin{tabular}{ccccc}
\hline Element & \multirow{2}{*}{ Code } & \multicolumn{3}{c}{ Coding level } \\
\cline { 3 - 5 } & & -1 & 0 & 1 \\
\hline Extraction temperature $\left[{ }^{\circ} \mathrm{C}\right]$ & $\mathrm{X}_{1}$ & 80 & 90 & 100 \\
Extraction time $[\mathrm{h}]$ & $\mathrm{X}_{2}$ & 2 & 4 & 6 \\
Ratio of material to liquid $[\mathrm{w}: \mathrm{v}]$ & $\mathrm{X}_{3}$ & 0.075 & 0.05 & 0.025 \\
\hline
\end{tabular}

Measurement of Crude Polysaccharide Content of Catathelasma Ventricosum Fruiting Body. Measurement of the reducing polysaccharide content in the extraction solution of Catathelasma ventricosum fruiting body:Fetch some appropriate concentration diluted extraction solution. Employ the DNS method to measure the reducing polysaccharide. (Add $2 \mathrm{~mL}$ DNS developing liquid. Develop in the boiling water for $10 \mathrm{~min}$. Cool down rapidly and terminate reaction. Measure the OD value at the location of $540 \mathrm{~nm}$. Calculate the content of the reducing polysaccharide through the comparison of corresponding glucose standard curves.)

Measurement of the total polysaccharide content in the extraction solution of Catathelasma ventricosum fruiting body:Fetch $1 \mathrm{~mL}$, the appropriate concentration diluted extraction solution of Catathelasma ventricosum, add $1 \mathrm{~mL}$ water, $1 \mathrm{~mL}$ phenol and $5 \mathrm{~mL}$ concentrated sulfuric acid in turn. Shake it evenly and put it in the boiling water bath for $15 \mathrm{~min}$. Quickly fetch it out, and cool it down in the ice-bath. At last, the ultraviolet spectrophotometer is used to test the absorption value at the location of $490 \mathrm{~mm}$. Calculate the total polysaccharide content by comparing the corresponding glucose standard curves.

Measurement of crude polysaccharide content in the extraction solution of Catathelasma ventricosum fruiting body: The calculation formula of the crude polysaccharide in the extraction solution: crude polysaccharides content=total polysaccharides content-reducing polysaccharides content. 
Measurement of in Vitro Biological Activity of Crude Polysaccharide in Catathelasma Ventricosum Fruiting Body. The experiment refers to relevant experiments conducted by LIU, et al. [10]

\section{Results and Discussion}

Optimization of the Crude Polysaccharide Extraction Process of Catathelasma Ventricosum Fruiting Body. RSA is a combination of Mathematics and Statistics. It is widely applied to the food industry. (Evaluate the influence of various variables on products so as to obtain the optimal conditions.) [11, 12] The advantage of RSA lies in that it can not only reduce the number of experiments and reduce the labor input, but also save time, while evaluating the function of multiple variables and their interaction. Generally speaking, a quadratic multi-term equation can be obtained according to corresponding experiment tables. The equation can be used to describe the influence of various variables on the response value, and the interaction between various variables can be determined.

The whole experiment design consists of 15 parallel experiments (including three central points) and is randomly distributed. Under different experiment conditions, the extraction amount of polysaccharides and various indexes of the biological activity are corresponding to Table 2 . According to the findings, ANOVA (Analysis of Variance) is conducted. After the quadratic RSA is analyzed, the one-degree term, the quadratic term and the interaction term of various variables (extraction temperature, extraction time and ratio of material to liquid) are corresponding to each response value.

Table 2 Experimental design and result of Box-Behnken

\begin{tabular}{|c|c|c|c|c|c|c|c|c|c|c|}
\hline \multirow{2}{*}{$\begin{array}{l}\text { Experiment } \\
\text { number }\end{array}$} & \multicolumn{3}{|c|}{ Variable [coding level] } & \multirow{2}{*}{$\mathrm{Y}_{1}$} & \multirow{2}{*}{$\mathrm{Y}_{2}$} & \multirow{2}{*}{$\mathrm{Y}_{3}$} & \multirow{2}{*}{$\mathrm{Y}_{4}$} & \multirow{2}{*}{$\mathrm{Y}_{5}$} & \multirow{2}{*}{$\mathrm{Y}_{6}$} & \multirow{2}{*}{$\mathrm{Y}_{7}$} \\
\hline & $\mathrm{X}_{1}$ & $\mathrm{X}_{2}$ & $\mathrm{X}_{3}$ & & & & & & & \\
\hline 1 & 0 & 1 & -1 & $7.53^{\mathrm{A}}$ & 2.84 & 2.52 & 91 & 2.89 & 1.19 & 4.97 \\
\hline 2 & 1 & 0 & -1 & 7.17 & 2.75 & 2.49 & 90 & 2.91 & 1.45 & 4.92 \\
\hline 3 & -1 & 0 & -1 & 6.40 & 2.86 & 2.53 & 89 & 2.44 & 1.23 & 4.97 \\
\hline 4 & 0 & 1 & 1 & 7.80 & 2.79 & 2.55 & 90 & 2.87 & 1.18 & 4.8 \\
\hline 5 & 0 & 0 & 0 & 8.03 & 2.81 & 2.51 & 87 & 2.43 & 0.92 & 4.92 \\
\hline 6 & 1 & 0 & 1 & 7.19 & 2.76 & 2.56 & 92 & 2.93 & 1.48 & 4.99 \\
\hline 7 & 0 & 0 & 0 & 8.05 & 2.80 & 2.49 & 89 & 2.42 & 0.91 & 4.93 \\
\hline 8 & -1 & 0 & 1 & 6.52 & 2.85 & 2.53 & 89 & 2.45 & 1.23 & 4.9 \\
\hline 9 & -1 & -1 & 0 & 6.31 & 2.77 & 2.50 & 90 & 2.44 & 1.19 & 5.03 \\
\hline 10 & -1 & 1 & 0 & 6.52 & 2.79 & 2.51 & 91 & 2.60 & 1.33 & 4.95 \\
\hline 11 & 0 & -1 & -1 & 7.10 & 2.83 & 2.55 & 89 & 2.49 & 0.97 & 4.95 \\
\hline 12 & 1 & -1 & 0 & 6.95 & 2.85 & 2.48 & 91 & 2.58 & 1.4 & 4.87 \\
\hline 13 & 0 & 0 & 0 & 8.06 & 2.80 & 2.52 & 88 & 2.42 & 0.93 & 4.97 \\
\hline 14 & 1 & 1 & 0 & 7.17 & 2.83 & 2.48 & 90 & 2.96 & 1.52 & 4.95 \\
\hline 15 & 0 & -1 & 1 & 7.68 & 2.88 & 2.54 & 91 & 2.52 & 0.99 & 4.96 \\
\hline
\end{tabular}

Note: ${ }^{\mathrm{A}}$ :Each value are represented as the average of three tests; $\mathrm{X}_{1}$ :extraction temperature $\left[{ }^{\circ} \mathrm{C}\right]$; $\mathrm{X}_{2}$ :extraction time $[\mathrm{h}] ; \mathrm{X}_{3}$ : ratio of material to liquid $[\mathrm{w}: \mathrm{v}] ; \mathrm{Y}_{1}:$ Polysaccharide extraction quantity $[\mathrm{g}]$; $\mathrm{Y}_{2}: \alpha$-glucosidase inhibitory activity $[\mu \mathrm{g} / \mathrm{mL}] ; \mathrm{Y}_{3}: \alpha$-amylase inhibitory activity $[\mu \mathrm{g} / \mathrm{mL}] ; \mathrm{Y}_{4}$ :AGEs inhibitory activity [\%]; $\mathrm{Y}_{5}: \mathrm{DPPH}$ scavenging activity $[\mu \mathrm{g} / \mathrm{mL}] ; \mathrm{Y}_{6}:$ Reducing power $[\mu \mathrm{g} / \mathrm{mL}]$; $\mathrm{Y}_{7}$ :Metal chelating activity $[\mu \mathrm{g} / \mathrm{mL}]$ 
Employment of RSA for the Optimization of the Polysaccharide Extraction Amount of Catathelasma Ventricosum Fruiting Body. The variance analysis results are shown in Table 3 . The significance of the regression model, $P<0.01$, suggests that the regression equation is extremely significant. $\mathrm{R}_{\text {Adj }}^{2}=96.38 \%$, this suggests that, among the nine factors in the whole regression equation, $96.38 \%$ of the variation distribution of the response value can be explained by the model. $36 \%$ of the total variation cannot be explained by the model. $\mathrm{R}^{2}=98.71 \%$, this suggests that there is a favorable fitting degree between the actually measured value and the predicted value. Generally speaking, the closer $\mathrm{R}^{2}$ value is to " 1 ", the closer the fitting model is in line with the actually measured data. [14] Therefore, the model can be used for the prediction of the practical situations of the polysaccharides extraction amount of Catathelasma ventricosum.

Table 3 Variance analysis of regression equation

\begin{tabular}{cccccc}
\hline Resource & $\begin{array}{c}\text { Degree of } \\
\text { freedom }\end{array}$ & Adj SS & Adj MS & Fvalue & Pvalue \\
\hline Regression model & 9 & 5.19815 & 0.57757 & 42.41 & $<0.001$ \\
One degree term & 3 & 1.17418 & 0.39139 & 28.74 & 0.001 \\
Quadratic term & 3 & 3.99742 & 1.33247 & 97.84 & $<0.001$ \\
Exchange term & 3 & 0.02655 & 0.00885 & 0.65 & 0.616 \\
Residual error & 5 & 0.06809 & 0.01362 & & \\
Lack of fit & 3 & 0.06763 & 0.02254 & 96.61 & 0.010 \\
Pure error & 2 & 0.00047 & 0.00023 & & \\
\hline
\end{tabular}

Note: $\mathrm{R}^{2}=98.71 \%, \mathrm{R}_{\text {Adj }}^{2}=96.38 \%$

From the significance test of the regression equation coefficient (Table 4), it can be seen that the linear effect of Factor $\mathrm{X}_{1}(P<0.01)$ on polysaccharide extraction amount of Catathelasma ventricosum is significant; the linear effect of Factor $\mathrm{X}_{2}$ and $\mathrm{X}_{3}(P=0.031$ 和0.030, $<0.05)$ on the polysaccharide extraction amount of Catathelasma ventricosum is significant; the hook face effect of Factor $\mathrm{X}_{1}^{2}(P<0.01)$ on polysaccharide extraction amount of Catathelasma ventricosum is extremely significant; the hook face effect of Factor $\mathrm{X}_{1}{ }^{2}$ and $\mathrm{X}_{3}{ }^{2}(P=0.004$ 和 $0.016,<0.05)$ on polysaccharide extraction amount of Catathelasma ventricosum is significant; while the interaction effect of interaction item factors including $\mathrm{X}_{1} \mathrm{X}_{2}, \mathrm{X}_{1} \mathrm{X}_{3}$ and $\mathrm{X}_{2} \mathrm{X}_{3}$, on polysaccharide extraction amount of Catathelasma ventricosum is not $\operatorname{significant}(P>0.05)$. After regression and fitting of various factors, the quadratic multiple regression equation of independent variables (including extraction temperature, extraction time and ratio of material to liquid) corresponding to different codes is obtained.

$$
Y=8.05+0,34 X_{1}+0.12 X_{2}+0.12 X_{3}-1.01 X_{1}^{2}-0.301 X_{2}^{2}-0.22 X_{3}^{2}
$$

Table 4 Estimated regression coefficients for polysaccharides extraction

\begin{tabular}{ccccc}
\hline Model item & $\begin{array}{c}\text { Regression } \\
\text { coefficient }\end{array}$ & $\begin{array}{c}\text { Standard error of } \\
\text { coefficient }\end{array}$ & T value & P value \\
\hline Constant & 8.04667 & 0.06738 & 119.430 & $<0.001$ \\
$\mathrm{X}_{1}$ & 0.34125 & 0.04126 & 8.271 & $<0.001$ \\
$\mathrm{X}_{2}$ & 0.12250 & 0.04126 & 2.969 & 0.031 \\
$\mathrm{X}_{3}$ & 0.12375 & 0.04126 & 2.999 & 0.030 \\
$\mathrm{X}_{1}{ }^{2}$ & -1.00833 & 0.06073 & -16.603 & $<0.001$ \\
$\mathrm{X}_{2}$ & -0.30083 & 0.06073 & -4.954 & 0.004 \\
$\mathrm{X}_{3}{ }^{2}$ & -0.21833 & 0.06073 & -3.595 & 0.016 \\
\hline
\end{tabular}




\begin{tabular}{lllll}
\hline $\mathrm{X}_{1} \mathrm{X}_{2}$ & 0.00250 & 0.05835 & 0.0430 & 0.967 \\
$\mathrm{X}_{1} \mathrm{X}_{3}$ & -0.02500 & 0.05835 & -0.428 & 0.686 \\
$\mathrm{X}_{2} \mathrm{X}_{3}$ & -0.07750 & 0.05835 & -1.328 & 0.242 \\
\hline
\end{tabular}

Note: $\mathrm{X}_{1}$ : extraction temperature $\left[{ }^{0} \mathrm{C}\right] ; \mathrm{X}_{2}$ : extraction time $[\mathrm{h}] ; \mathrm{X}_{3}$ : ratio of material to liquid [w:v]

According to regression analysis results in Table 4, the partial derivative of the fitting equation is conducted so as to obtain the maximum predicted value of the polysaccharide extraction amount of Catathelasma ventricosum. In other words, when the extraction temperature is $91.72{ }^{\circ} \mathrm{C}$, the extraction time is $4.34 \mathrm{~h}$ and the ratio of material to ratio is 0.05 , the predicted value of the polysaccharide extraction amount of Catathelasma ventricosum is $8.10 \mathrm{~g}$. (From $25 \mathrm{~g}$ of freeze-dried powder raw material)

Employment of RSA for the Optimization of Polysaccharide DPPH Scavenging Activity of Catathelasma Ventricosum. The ANOVA results are shown in Table 5. The regression model $(P=0.008)$ shows that the regression equation is extremely significant. $\mathrm{R}^{2}{ }_{\mathrm{Adj}}=87.06 \%$, suggesting that among the nine factors in the whole regression equation, the variation distribution of $87.06 \%$ of response values can be explained by the model; while only the variation distribution of $12.9 \%$ of the total cannot be explained by the model. (Generally speaking, $\mathrm{R}_{\text {Adj }}^{2}>80 \%$ means acceptability.) $\mathrm{R}^{2}=95.38 \%$, suggesting that there is a good fitting degree between the actually measured value and the predicted value of the polysaccharide DPPH scavenging activity of Catathelasma ventricosum. Therefore, the model can be used to predict the practical situation of polysaccharide DPPH scavenging activity of Catathelasma ventricosum.

Table 5 Variance analysis of regression equation

\begin{tabular}{cccccc}
\hline Resource & $\begin{array}{c}\text { Degree of } \\
\text { freedom }\end{array}$ & Adj SS & Adj MS & $F$ value & P value \\
\hline Regression model & 9 & 0.638792 & 0.070977 & 11.47 & 0.008 \\
One degree term & 3 & 0.471025 & 0.157008 & 25.37 & 0.002 \\
Quadratic term & 3 & 0.155017 & 0.051672 & 8.35 & 0.022 \\
Exchange term & 3 & 0.012750 & 0.004250 & 0.69 & 0.598 \\
Residual error & 5 & 0.030942 & 0.006188 & & \\
Lack of fit & 3 & 0.030875 & 0.010292 & 308.75 & 0.003 \\
Pure error & 2 & 0.000067 & 0.000033 & & \\
\hline
\end{tabular}

Note: $\mathrm{R}^{2}=95.38 \%, \mathrm{R}_{\text {Adj }}^{2}=87.06 \%$

From the significance test of the coefficients of the regression equation (Table 6), it can be seen that the linear effect of the one-degree item of $X_{1}$ and $X_{2}$ on polysaccharide DPPH scavenging activity is extremely significant; while the $P$ value of the one-degree term of $\mathrm{X}_{3}(P>0.05)$, which suggests its linear effect on the response value is not significant. The $P$ value of the quadratic terms, including $\mathrm{X}_{1}^{2}, \mathrm{X}_{2}^{2}$ and $\mathrm{X}_{3}^{2}$, is $0.049,0.037$ and $0.013(P<0.05)$, respectively. This suggests that the curve effect of quadratic terms on polysaccharide DPPH scavenging activity of Catathelasma ventricosum is significant; while the interaction effect of interaction factors, including $\mathrm{X}_{1} \mathrm{X}_{2}, \mathrm{X}_{1} \mathrm{X}_{3}$ and $\mathrm{X}_{2} \mathrm{X}_{3}$, on polysaccharide DPPH scavenging activity of Catathelasma ventricosum is not obvious $(P>0.05)$. After the regression fitting of various factors, the quadratic multiple regression function of independent variables (extraction temperature, time and ratio of material to liquid) corresponding to each code can be obtained:

$$
Y=2.42+0.18 X_{1}+0.16 X_{2}+0.05 X_{1}^{2}-0.04 X_{2}^{2}-0.01 X_{3}^{2}
$$


Table 6 Estimated regression coefficients for DPPH radicals are scavenged [mg/mL]

\begin{tabular}{ccccc}
\hline Model item & $\begin{array}{c}\text { Regression } \\
\text { coefficient }\end{array}$ & $\begin{array}{c}\text { Standard error of } \\
\text { coefficient }\end{array}$ & T value & Pvalue \\
\hline Constant & 2.42333 & 0.04542 & 53.356 & $<0.001$ \\
$\mathrm{X}_{1}$ & 0.18125 & 0.02781 & 6.517 & 0.001 \\
$\mathrm{X}_{2}$ & 0.16125 & 0.02781 & 5.798 & 0.002 \\
$\mathrm{X}_{3}$ & 0.00500 & 0.02781 & 0.180 & 0.864 \\
$\mathrm{X}_{1}{ }^{2}$ & 0.10583 & 0.04094 & 2.585 & 0.049 \\
$\mathrm{X}_{2}$ & 0.11583 & 0.04094 & 2.829 & 0.037 \\
$\mathrm{X}_{3}{ }^{2}$ & 0.15333 & 0.04094 & 3.745 & 0.013 \\
$\mathrm{X}_{1} \mathrm{X}_{2}$ & 0.05500 & 0.03933 & 1.398 & 0.221 \\
$\mathrm{X}_{1} \mathrm{X}_{3}$ & 0.00250 & 0.03933 & 0.064 & 0.952 \\
$\mathrm{X}_{2} \mathrm{X}_{3}$ & -0.01250 & 0.03933 & -0.318 & 0.763 \\
\hline
\end{tabular}

Note: $\mathrm{X}_{1}$ : extraction temperature $\left[{ }^{\circ} \mathrm{C}\right] ; \mathrm{X}_{2}$ : extraction time $[\mathrm{h}] ; \mathrm{X}_{3}$ : ratio of material to liquid [w:v]

According to the regression analysis results in Table 6, the partial derivative of the fitting equation is worked out so as to obtain the maximum predicted value of the polysaccharide DPPH scavenging activity of Catathelasma ventricosum fruiting body. In other words, when the extraction temperature is $84.83{ }^{\circ} \mathrm{C}$ and the extraction time is $2.93 \mathrm{~h}$, the predicted value of the polysaccharide DPPH scavenging activity of Catathelasma ventricosum is $2.32 \mu \mathrm{g} / \mathrm{mL}$.

Employment of RSA to Optimize the Polysaccharide Reducing Power of Catathelasma Ventricosum. The ANOVA results are shown in Table 7, the regression model, $P<0.001$, suggests that the regression function is extremely significant. $\mathrm{R}_{\text {Adj }}^{2}=98.38 \%$, suggesting that, among the nine factors in the whole factors, the variation distribution of $98.38 \%$ of response value can be explained by the model, while the variation distribution of $1.62 \%$ of the total variation cannot be explained by the model. $\mathrm{R}^{2}=99.42 \%$, suggesting that there is a good fitting degree between the actually measured value and the predicted value of the polysaccharide reducing power of Catathelasma ventricosum. Therefore, the model can be applied to the prediction of the practical situation of the polysaccharides.

Table 7 Variance analysis of regression equation

\begin{tabular}{cccccc}
\hline Resource & $\begin{array}{c}\text { Degree of } \\
\text { freedom }\end{array}$ & Adj SS & Adj MS & F value & P value \\
\hline Regression model & 9 & 0.630898 & 0.070100 & 95.37 & $<0.001$ \\
One degree term & 3 & 0.150925 & 0.050308 & 68.45 & $<0.001$ \\
Quadratic term & 3 & 0.479423 & 0.159808 & 217.43 & $<0.001$ \\
Exchange term & 3 & 0.000550 & 0.000183 & 0.25 & 0.859 \\
Residual error & 5 & 0.003675 & 0.000735 & & \\
Lack of fit & 3 & 0.003475 & 0.001158 & 11.58 & 0.081 \\
Pure error & 2 & 0.000200 & 0.000100 & & \\
\hline
\end{tabular}

Note: $\mathrm{R}^{2}=99.42 \%, \mathrm{R}_{\text {Adj }}^{2}=98.38 \%$

From the significance test of the regression function coefficient(Table 8), it can be seen that the linear effect of the one-degree term factor, $\mathrm{X}_{1}$ and $\mathrm{X}_{2} \quad(P<0.01)$, on the polysaccharide reducing power of Catathelasma ventricosum is extremely significant; while the $P$ value of $\mathrm{X}_{3}$ is $0.624(P>0.05)$, suggesting insignificant linear effect on the response value; the $P$ value of $\mathrm{X}_{1}^{2}, \mathrm{X}_{2}{ }^{2}$ and $\mathrm{X}_{3}{ }^{2}$ of the quadratic term is $<0.001,0.002$ and $0.003(\mathrm{P}<0.01)$, respectively. This suggests the curve effect of the quadratic terms on the polysaccharide reducing power of Catathelasma ventricosum is extremely 
signficiant. However, the interaction effect of the interaction factors, including $\mathrm{X}_{1} \mathrm{X}_{2}, \mathrm{X}_{1} \mathrm{X}_{3}$ and $\mathrm{X}_{2} \mathrm{X}_{3}$, on the polysaccharide reducing power of Catathelasma ventricosum is not significant $(P>0.05)$. After regression and fitting of various factors, the quadratic multiple regression equation of the independent variables (including extraction temperature, extraction time and ratio of material to liquid) corresponding to each code is obtained:

$$
Y=0.92+0.11 X_{1}+0.08 X_{2}+0.35 X_{1}^{2}+0.09 X_{2}^{2}+0.08 X_{3}^{2}
$$

Table 8 Estimated regression coefficients for reducing power [mg/mL]

\begin{tabular}{ccccc}
\hline Model item & $\begin{array}{c}\text { Regression } \\
\text { coefficient }\end{array}$ & $\begin{array}{c}\text { Standard error of } \\
\text { coefficient }\end{array}$ & T value & P value \\
\hline Constant & 0.920000 & 0.015652 & 58.777 & $<0.001$ \\
$\mathrm{X}_{1}$ & 0.108750 & 0.009585 & 11.346 & $<0.001$ \\
$\mathrm{X}_{2}$ & 0.083750 & 0.009585 & 8.737 & $<0.001$ \\
$\mathrm{X}_{3}$ & 0.005000 & 0.009585 & 0.522 & 0.624 \\
$\mathrm{X}_{1}{ }^{2}$ & 0.352500 & 0.014109 & 24.984 & $<0.001$ \\
$\mathrm{X}_{2}{ }^{2}$ & 0.087500 & 0.014109 & 6.202 & 0.002 \\
$\mathrm{X}_{3}$ & 0.075000 & 0.014109 & 5.316 & 0.003 \\
$\mathrm{X}_{1} \mathrm{X}_{2}$ & -0.005000 & 0.013555 & -0.369 & 0.727 \\
$\mathrm{X}_{1} \mathrm{X}_{3}$ & 0.007500 & 0.013555 & 0.553 & 0.604 \\
$\mathrm{X}_{2} \mathrm{X}_{3}$ & -0.007500 & 0.013555 & -0.553 & 0.604 \\
\hline
\end{tabular}

Note: $\mathrm{X}_{1}$ : extraction temperature $\left[{ }^{\circ} \mathrm{C}\right] ; \mathrm{X}_{2}$ : extraction time $[\mathrm{h}] ; \mathrm{X}_{3}$ : ratio of material to liquid $[\mathrm{w}: \mathrm{v}]$

According to regression analysis results in Table 8, the partial derivative of the fitting equation is worked out so as to obtain the maximum prediction value of the polysaccharide reducing power of Catathelasma ventricosum. In other words, when the extraction temperature is $85.47^{\circ} \mathrm{C}$ and the extraction time is $3.01 \mathrm{~h}$, the predicted value of the polysaccharide reducing power of Catathelasma ventricosum is $0.89(\mu \mathrm{g} / \mathrm{mL})$.

Employment of RSA to Optimize the Other Response Value of Polysaccharides of Catathelasma ventricosum. According to Table 2, it can be seen that the extraction temperature, extraction time and ratio of material to liquid have no significant influence on the inhibitory activity of $\alpha$-glucosaccharase, $\alpha$-amylase, AGEs and metal-chelating. Therefore, when the optimal extraction conditions are defined, the influence of extraction conditions on them is no longer considered.

Determination and Verification of Optimal Polysaccharide Extraction Conditions of Catathelasma ventricosum. According to the above regression analysis results and in view of the extraction efficiency, the principle of chosing the extraction method with the highest biological activity. At last, the optimal polysaccharide extraction conditions of Catathelasma ventricosum can be determined: extraction temperature: $85^{\circ} \mathrm{C}$; extraction time: $3 \mathrm{~h}$; ratio of material to liquid: $0.05 \mu \mathrm{g} / \mathrm{mL}$.

Based on the above conditions, the predicted value and the actually measured value of various response values (in Table 9) are obtained. From the results, it can be seen that the actually measured value and the predicted value of various response values (polysaccharide extraction amount, DPPH scavenging activity and reducing power) are close to each other. This suggests that the regression function can authentically and efficiently reflect the influence of various factors (including extraction temperature, extraction time and ratio of material to liquid) and that the employment of RSA to optimize polysaccharide extraction conditions of Catathelasma ventricosum is feasible and the regression model is very reliable. 
Table 9 The results of experimental verification

\begin{tabular}{cccccccccccccc}
\hline $\mathrm{Y}_{1}$ & $\mathrm{y}_{1}$ & $\mathrm{Y}_{2}$ & $\mathrm{y}_{2}$ & $\mathrm{Y}_{3}$ & $\mathrm{y}_{3}$ & $\mathrm{Y}_{4}$ & $\mathrm{y}_{4}$ & $\mathrm{Y}_{5}$ & $\mathrm{y}_{5}$ & $\mathrm{Y}_{6}$ & $\mathrm{y}_{6}$ & $\mathrm{Y}_{7}$ & $\mathrm{y}_{7}$ \\
\hline $7.59^{\mathrm{A}}$ & 7.53 & 2.85 & $\mathrm{ND}^{\mathrm{B}}$ & 2.57 & $\mathrm{ND}$ & 90 & $\mathrm{ND}$ & 2.31 & 2.29 & 0.87 & 0.88 & 4.96 & $\mathrm{ND}$ \\
\hline
\end{tabular}

Note: ${ }^{\mathrm{A}}$ :Each value are represented as the average of three tests; $\mathrm{X}_{1}$ :extraction temperature $\left[{ }^{0} \mathrm{C}\right]$; $\mathrm{X}_{2}$ :extraction time $[\mathrm{h}] ; \mathrm{X}_{3}$ :ratio of material and liquid $[\mathrm{w}: \mathrm{v}] ; \mathrm{Y}_{1}:$ Polysaccharide extraction quantity $[\mathrm{g}] ; \mathrm{Y}_{2}: \alpha$-glucosidase inhibitory activity $[\mu \mathrm{g} / \mathrm{mL}] ; \mathrm{Y}_{3}: \alpha$-amylase inhibitory activity $[\mu \mathrm{g} / \mathrm{mL}]$; $\mathrm{Y}_{4}$ :AGEs inhibitory activity [\%]; $\mathrm{Y}_{5}: \mathrm{DPPH}$ scavenging activity $[\mu \mathrm{g} / \mathrm{mL}] ; \mathrm{Y}_{6}:$ Reducing power $[\mu \mathrm{g} / \mathrm{mL}] ; Y_{7}:$ Metal chelating activity $[\mu \mathrm{g} / \mathrm{mL}]$. All lowercase letter " $\mathrm{y}$ " is their predictive value.

\section{Acknowledgements}

This work was supported by Scientific Research Foundation of Sichuan Agricultural University (No. 06021400) and The National Natural Science Foundation of China (No. 31501550) .

\section{References}

[1]Y.C. Dai, L.W. Zhou and Z.L. Yang, A revised checklist of edible fungi in China, Mycosystema. 29 (2010) 1-21.(In Chinese)

[2]X.L. Luan and D. Jiang, The name of the important edible fungus analysis in China, Edible and medicinal mushrooms. 20(2012)195-201. (In Chinese)

[3]Z. Li, J. Chen, M. Wang and Q. Luo, Parts of physical and chemical properties of the ploysaccharides from Catathelasma ventricosum (Pk.) Sing. Armillaria ventricosa Peck and their inhibitory effect on S-180 tumor, Microbiology.China. 40(2013). (In Chinese)

[4]J.X. Sun, Y.F. Wang, J.H. Wang, L. Shi, Z.T. Liu and C.L. Pan, Research advances on ploysaccharide extracting technology of edible fungi, Edible fungi of China. 31(2012)6-9.

[5]S.W. Cui, Ploysaccharide gums from agriculture products:Processing, structure and functionality, Lancaster:Technomic Publishing, 2001.

[6]Y. Sun, J. Tang and X. Gu, Water -soluble polysaccharides from Angelica sinensis

(Oliv.)Diels:Preparation, characterization and bioactivity, International Journal of Biological

Macromolecules, 36( 2005)283-289.

[7]Q.X. Zhang, P.J. Yang and G.H. Yang, Preparation of acid-extraction methods and propreties of soy hull pectin, Food Research and Development. 26(2005)40-43. (In Chinese)

[8]P. Somboonpanyakul, Q. Wang and W. Cui, Malva nut gum(Part I):Extraction and physicochemical characterization, Carbohydrate Polymers. 64(2006)247-253.

[9]R.C. Sun, S. Hughes, Fractional isoation and physico-chemical characterization of alkali-soluble polysaccharides from sugar beet pulp, Carbohydrate Polymers. 38(1999)273-281.

[10]Y.T. Liu, J. Sun, Z.Y. Luo and S.Q. Rao, Chemical composition of five wild edible mushrooms collected from Southwest China and their antihyperglycemic and antioxidant activity, Food Chemical Toxicology. 50(2012)1238-1244.

[11]W. Cui, G. Mazza, B.D. Oomah and C.G. Biliaderis, Optimization of an Aqueous extraction process for flaxseed gum by response surface methodology, LWT-Food Science and Technology. 27(1994)363-369.

[12]M.S. Tanyildizi, D. Ozer and M. Elibol, Optimization of $\alpha$-amylase production by Bacillus sp. Using response surface methodology, Process Biochemistry. 40(2005)2291-2296.

[13]Y.W. Wu, Experimental design and data analysis, Suzhou: Suzhou University Press, 2002.(In Chinese)

[14]W.C. Lee, S. Yusof and N.S.A. Hamid, Optimizing conditions for enzymatic clarification of banana juice using response surface methodology (RSM), Journal of Food Engineering. 73(2006)55-63. 